\title{
Positive effects of a qigong and aerobic exercise program on physical health in elderly Japanese women: an exploratory study
}

\author{
Takeshi Sakata $\cdot$ Qiming Li $\cdot$ Michio Tanaka $\cdot$ \\ Fumihiro Tajima
}

Received: 10 January 2007 / Accepted: 27 December 2007/Published online: 29 March 2008

(C) The Japanese Society for Hygiene 2008

\begin{abstract}
Objective To determine the effects of a 12-week qigong and aerobic exercise program on the physical well-being of relatively healthy elderly Japanese women.

Methods In the first study, 72 elderly Japanese women who were relatively healthy and naive to qigong completed the 12-week qigong and aerobic exercise program. Physical function, body composition, and abdominal fat were evaluated. In the second study, we examined the effects of qigong alone on physical function. Twenty-nine participants in each of two groups (divided according to their residences) underwent a 12-week program: qigong and aerobic exercise (residents in Yura Town) or qigong exercise alone (residents in Mihama Town).

Results In the first study, physical function including lung capacity, trunk bending, normal walking for $30 \mathrm{~m}$, and rising from a supine position significantly improved after the 12-week program. In addition, body fat diminished significantly during the program. In the second study, both exercise programs ("qigong and aerobic" and "qigong alone") similarly increased walking speed (normal and maximum walking) and rising speed.

Conclusions The findings of this exploratory study demonstrated that a 12-week qigong and aerobic exercise
\end{abstract}

T. Sakata $(\bowtie)$

Health Support Center Kitade, 733-1 Takara,

Yukawa-cho, Gobo, Wakayama 644-0011, Japan

e-mail: sakatatakeshi@hotmail.com

Q. Li · M. Tanaka

Medical and Fitness Aquo, Gobo, Japan

F. Tajima

Department of Rehabilitation Medicine, Wakayama Medical

University School of Medicine, Wakayama, Japan program was associated with improvements in physical function and a reduction in body fat. The qigong exercise program alone positively influenced physical function. The qigong program appears to be an appealing means of improving the physical health of elderly persons.

Keywords Qigong - Exercise - Physical function · Body fat · Elderly women

\section{Introduction}

Qigong (che-gong), a traditional Asian training method, combines aerobic, isometric, and isotonic elements with meditation and relaxation. The physiological and psychosomatic effects of static training in the qigong program have been studied; specifically, "Qi" flows in certain directions and is dependent on deep breathing, posture, and meditation [1-7]. There is evidence to suggest that qigong is useful for the treatment of psychosomatic and stress-related disorders $[1,2]$. Furthermore, qigong has been shown to effectively reduce blood pressure and catecholamine levels in patients with essential hypertension [3]. Other studies have shown that qigong modulates the secretion levels of thyroid hormones, parathyroid hormone, growth hormone, and testosterone in the elderly $[4,5]$, and affects the profile of lymphocyte subsets in human peripheral blood [6].

Unlike static qigong training, dynamic qigong training is characterized by slow whole-body performance with natural breathing and maintenance of full force. Floor 6-style qigong and Shaolin internal qigong are forms of dynamic qigong training; the former is based on six kinds of performance in a supine position, and the later is based on several kinds of performance, special postures, and motion in a standing position. Physiologically, Shaolin internal qigong 
reportedly improves respiratory efficiency in skilled subjects without affecting the heart rate or maximum oxygen uptake levels in both skilled and unskilled subjects [8].

Physical activity programs improve functional status, health, and quality of life in the elderly [9-16]. Moderateintensity exercise intervention can reduce the incidence of falls due to improved physical function in elderly Japanese women [9]. Higher than normal body fat is associated with obesity and abdominal fat. Trunk fat mass is a strong predictor of insulin resistance and dyslipidemia in postmenopausal women [17]. An enlarged waist combined with elevated triglycerides is a strong predictor of cardiovascular risk in postmenopausal women [18]. However, regular aerobic exercise such as brisk walking reduces body weight and body fat in overweight postmenopausal women [10].

The majority of physical activity programs designed for the elderly are of limited intensity, ranging from low to moderate-intensity, to avoid the risk of musculoskeletal injury and cardiovascular problems [9-13].

We hypothesized that an exercise program mainly composed of Floor 6-style qigong and Shaolin internal qigong would be safe and would improve physical health of the elderly because of the unique training style: slow whole-body performance with natural breathing and full force maintenance. To our knowledge, no published studies have examined the effects of the qigong exercise program on the physical health of the elderly.

In this exploratory study, we first examined whether a 12-week qigong and aerobic exercise program improves physical health in elderly Japanese women by focusing on physical function and body fat parameters that would be expected to demonstrate any positive effects $[9,10]$ of an exercise program in elderly subjects. Aging induces a decline in physiological status and a lack of response to various forms of training. Thus, the participants were divided by age into a 60-69 year group and a $\geq 70$ year group.

In the first study, the exercise program demonstrated positive changes on physical health in the elderly women. However, it was unclear how much each exercise ("qigong" or "aerobic") was associated with the positive changes during the 12-week program. Therefore, in the second study, we examined whether a 12-week program of qigong exercise alone would influence physical function in elderly Japanese women.

\section{Methods}

Subjects and qigong exercise programs

In the first study (in 2003), elderly women between 60-86 years old living in Yura Town, Wakayama, Japan, who were relatively healthy and naive to qigong were recruited through advertisements at a fitness center (Aquo) and various public centers. Eligibility to participate in the study was determined by screening questionnaires and physical examination. Exclusion criteria included major medical illnesses and orthopedic problems that could hamper participation in a qigong and aerobic exercise program. Signed informed consent for the study was obtained from each subject. Eighty elderly women were recruited and five were excluded under the exclusion criteria above. Seventy-five participants were thus enrolled in the qigong and aerobic exercise program; 72 completed the 12-week course. Three enrollees discontinued for personal reasons, not related to physical condition, and their data were not included in the analysis.

Participants attended one 90-min qigong exercise program (45-min qigong exercise, a 20-min qigong lecture and rest, and an optional 25-min of walking in a swimming pool or ergometer exercise) each week, and were asked to complete a minimum of $20 \mathrm{~min}$ daily qigong practice at home except for the day of the group session (six times/week). Each group was instructed by well-trained qigong instructors. The program included a standard Floor 6-style qigong and Shaolin internal qigong. Floor 6-style qigong was performed in a supine position on a flat mat and included breathing impurity-out (deep breathing), breathing purity-in (pelvic off with deep breathing), and elevating buttocks, bending knees, rotating legs, and bending ankles activities. Shaolin internal qigong was preformed in a standing position on a flat mat and included Shaolin standing Zen, pushing eight horses forward, king holding-up a tripod, opening and closing arms, bow-shaped pose, and pushing eight horses forward with bow-shaped pose. The participants could choose any performances (postures) from Floor 6-style qigong and/or Shaolin internal qigong for their minimum of $20 \mathrm{~min}$ of daily qigong practice. They received detailed and illustrated instructions for home practice, and completed daily logs to monitor the frequency and duration of practice. The participants optionally performed walking in a swimming pool or an ergometer exercise in each class session. The intensities of the optional exercises were at comfortable levels for each participant.

Anthropometric measures

On the initial day of the 12-week exercise program, heights and weights of the participants were measured with a stadiometer and a calibrated balance scale. Body mass index (BMI) was calculated as weight divided by the square of height in meters $\left(\mathrm{kg} / \mathrm{m}^{2}\right)$.

The study participants underwent evaluations of physical functions, body composition (bone mineral content 
(BMC), body fat, soft tissue lean mass), and subcutaneous and visceral fat in divided subgroups (each subgroup: 10-15 participants) during the week before and the week after the 12-week exercise program.

\section{Physical functions}

The tests selected (lung capacity, reaction time, flexibility, walking ability, and balance) were based on previously reported findings [9, 19], with some modifications. In brief, lung capacity $(\mathrm{ml})$ was measured using a spirometer (Chestgraph Jr. HI-101; Chest Inc., Japan). Whole-body reaction was measured in seconds (s); the participant hopped from a board as soon as possible when the signal light was turned on. Duration of standing (s) using a preferred leg with open eyes was measured for stork stand. Trunk bending $(\mathrm{cm})$ with maximal effort in the sitting position was measured as the distance between the finger tips and a fixed line on the table. Walking speed was measured by counting the time in seconds to walk a $30-\mathrm{m}$ flat path at normal speed (normal walking for $30 \mathrm{~m}$; s) or maximum speed (maximum walking for $30 \mathrm{~m}$; s). Rising (s) represented the time in seconds that participants needed to stand upright from the supine position.

\section{Body composition}

Subtotal and regional body composition (bone mineral content, body fat, and soft tissue lean mass for trunk, arms, and legs) were determined by dual energy X-ray absorptiometry (DXA) using a Hologic QDR-4500A (software version 11.2; Hologic, Waltham, MA, USA). The standard procedures recommended by the manufacturer were followed to define trunk, arm, and leg regions.

\section{Subcutaneous and visceral fat}

Subcutaneous and visceral fat in the abdomen were measured using a Helical CT scan (Xvision SP; Toshiba, Japan). Subjects were asked to lie in the supine position with their arms above their head and their legs slightly bent. A single scan $(10 \mathrm{~mm})$ of the abdomen was taken at the level of the L4-L5 vertebrae and analyzed for the cross-sectional area of adipose tissue, which was then expressed in $\mathrm{cm}^{2}$. Areas were calculated by multiplying the number of pixels of a given tissue type by the pixel number (pixel density).

\section{Second study}

In the second study (in 2005), elderly women between 64-71 years of age living in Yura and Mihama Towns,
Wakayama, who were relatively healthy and naive to qigong were recruited in the same way as in the first study, including eligibility, exclusion criteria, and signed informed consent. Thirty elderly women were recruited (none was excluded) from each town. Participants were divided into two groups according to their residences: a qigong and aerobic group (residents in Yura Town) and a qigong group (residents in Mihama Town). The qigong and aerobic group enrolled in a 12-week qigong and aerobic exercise program that was the same as in the first study. However, optional aerobic exercise was not offered as an option for the qigong group (that is, qigong exercise was undertaken alone). In each group, 29 completed the 12-week course. Two enrollees (one in each group) discontinued for personal reasons, not related to physical condition, and their data were not included in the analysis. The second study participants underwent the same evaluations of physical functions (walking ability and rising) as those in the first study.

The studies (the first study and second study) were performed before the establishment of the Ethics Committee in Kitade hospital. Therefore the publication of results in the studies was approved by the Ethics Committee of Kitade hospital.

Statistical analysis

Data are presented as mean \pm standard deviation (SD). Paired Wilcoxon tests were used for analyzing the effects before and after the 12-week exercise programs. Wilcoxon-Mann-Whitney tests were also used for evaluating the differences between the two age groups ("60-69 years" and "over 70 years") or exercise programs ("qigong and aerobic" and "qigong alone") at baseline or post-intervention. Two-way repeated-measures analysis of variance was used for evaluating the interaction between groups ("qigong and aerobic" and "qigong alone") and time ("baseline" and "post") in the second study. All data were analyzed using KaleidaGraph 3.6.4 software (Synergy Software, PA, USA). Statistical significance was stated for $P$ values $<0.05$.

\section{Results}

First study

In the first study, 72 participants aged 60-86 years $(70.9 \pm 4.8)$ completed the study. The participants were divided by age into a 60-69 year group (67.0 \pm 1.9 , $n=35)$ and $\mathrm{a} \geq 70$ year group $(74.3 \pm 3.9, n=37)$. The anthropometric features of the participants are listed in 
Table 1 Anthropometry of the first study participants

\begin{tabular}{lrrr}
\hline & $\begin{array}{l}60-69 \text { years } \\
(n=35)\end{array}$ & $\begin{array}{l}\geq 70 \text { years } \\
(n=37)\end{array}$ & \multicolumn{1}{l}{$\begin{array}{l}\text { Total } \\
(n=72)\end{array}$} \\
\hline Height $(\mathrm{cm})$ & $151.5 \pm 4.9$ & $151.5 \pm 4.7$ & $151.5 \pm 4.7$ \\
Weight $(\mathrm{kg})$ & $56 \pm 8.4$ & $54.2 \pm 8.1$ & $55 \pm 8.2$ \\
Body mass index $\left(\mathrm{kg} / \mathrm{m}^{2}\right)$ & $24.4 \pm 3.2$ & $23.6 \pm 3.3$ & $24.0 \pm 3.2$ \\
\hline
\end{tabular}

Data are means $\pm \mathrm{SD}$

Table 1. There were no significant differences in heights, weights, and BMIs between the two groups at baseline.

The 12-week qigong and aerobic exercise program significantly increased physical functions, as measured by lung capacity, stork stand, trunk bending, walking speed (normal walking for $30 \mathrm{~m}$ ), and rising speed (Table 2). The exercise program significantly decreased body composition parameters of fat mass in the trunk, legs, and subtotal body. The program also significantly increased lean soft tissue mass in the trunk and subtotal body, while it did not alter the BMC in the trunk, arms, legs, or subtotal body during the 12 weeks. The 12 -week program significantly decreased subcutaneous fat, but not visceral fat, in the abdomen.

The $\geq 70$ year group had significantly lower measurements for stork stand, maximum walking speed, rising speed, and BMC of arms, legs, and subtotal body than the 60-69 year group at baseline. The $\geq 70$ year group had significantly lower lung capacity, maximum walking speed, and BMC of arms, legs, and subtotal body than the 60-69 year group at the post-intervention measurement. Unlike the 60-69 year group, the 12-week program did not significantly alter walking speed, fat mass in the trunk, lean soft tissue mass in the trunk and subtotal body, and subcutaneous fat of the abdomen in the $\geq 70$ year group. It significantly increased stork stand in the $\geq 70$ year group, but not in the 60-69 year group.

\section{Second study}

In the first study, the 12-week qigong and aerobic exercise program induced positive changes in the elderly women. We then performed the second study to examine whether a 12-week program of qigong exercise alone would positively influence physical function in elderly women. In this second study, we evaluated walking ability and rising, which are important for daily living activities in the elderly. There were no significant differences in ages, heights, weights, and BMIs (Table 3), and in walking and rising speeds (Table 4), between the two groups at baseline. Both exercise programs ("qigong and aerobic" and "qigong alone") significantly increased walking speed (normal and maximum walking) and rising speed. There were no significant differences in walking and rising speeds between the two groups at post-intervention. There were no significant interactions between groups and time in the second study (Table 4). Therefore, it was considered that at least the positive changes on physical function in the first study were associated with qigong exercise alone.

\section{Discussion}

The results of this exploratory study indicated that a 12 -week qigong and aerobic exercise program was associated with improvements in physical function and a reduction of body fat in elderly Japanese women.

Tai Chi, a traditional Chinese physical exercise, is known to improve physical function and reduce the risk of falling in the elderly [12]. Tai Chi originated from an ancient Chinese martial art consisting of a series of slow but continuous movements of every body part. Investigation of the effects of Tai Chi on physical health indicated that the most popular style among the many Tai Chi styles is the Yang style. [12, 13] Yang style demands constant knee flexion, wide stance width, and a steady slow speed.

Qigong is similar to Tai Chi training but has a different style of performance. Qigong originated from a self-management healthcare technique and combines aerobics, isometrics, isotonics, meditation, and relaxation. In our study, dynamic qigong training (Floor 6-style and Shaolin internal qigong) was used as the qigong practice. Unlike Tai Chi training, qigong practice does not always involve a series of movements conducted in a standing position. Therefore, trainees can choose and perform each movement or posture independently. Each performance in qigong practice is much slower and simpler than Tai Chi. Therefore, we believe qigong practice is suitable exercise for beginners or appropriate low level physical activity for the elderly.

Both osteoporosis and falls cause serious problems associated with femoral neck and vertebral body fractures. A regular exercise intervention program and a Tai Chi program reduce the risk of falls mainly by enhancing physical function in the elderly $[9,12]$. The 12-week program here positively influenced physical function, such as walking ability, balance, and rising. Therefore, the qigong and aerobic exercise program might prevent falls in the elderly.

In the elderly, aging decreases physiological status and response to various forms of training. However, regular exercise improves physical activities in those over 70 years of age [9]. Our finding that qigong and aerobic exercise significantly improved physical function in elderly individuals over 70 years of age is supported by previous results. 
Table 2 Effects of 12-week qigong with aerobic exercise program on physical function, body composition and abdominal fats

\begin{tabular}{|c|c|c|c|c|c|c|c|}
\hline & & \multicolumn{2}{|c|}{$60-69$ years $(n=35)$} & \multicolumn{2}{|c|}{$\geq 70$ years $(n=37)$} & \multicolumn{2}{|l|}{ Total $(n=72)$} \\
\hline & & Baseline & Post-intervention & Baseline & Post-intervention & Baseline & Post-intervention \\
\hline \multicolumn{2}{|l|}{ Lung capacity (ml) } & $2,032.7 \pm 581.2$ & $2,202.5 \pm 541.8^{*}$ & $1,904.2 \pm 478.3$ & $1,940.5 \pm 469.7 * \#$ & $1,992.3 \pm 476.0$ & $2,092.2 \pm 456.2^{*}$ \\
\hline \multicolumn{2}{|l|}{$\begin{array}{l}\text { Whole-body } \\
\text { reaction (s) }\end{array}$} & $0.411 \pm 0.050$ & $0.429 \pm 0.064$ & $0.448 \pm 0.081$ & $0.444 \pm 0.066$ & $0.431 \pm 0.070$ & $0.437 \pm 0.065$ \\
\hline \multicolumn{2}{|l|}{$\begin{array}{l}\text { Stork stand (eyes } \\
\text { open) (s) }\end{array}$} & $55.0 \pm 33.8$ & $57.6 \pm 33.2$ & $35.4 \pm 32.5 \#$ & $46.5 \pm 32.3^{*}$ & $44.7 \pm 34.5$ & $52.1 \pm 33.0^{*}$ \\
\hline \multicolumn{2}{|l|}{$\begin{array}{l}\text { Trunk bending } \\
(\mathrm{cm})\end{array}$} & $15.9 \pm 7.1$ & $22.1 \pm 6.4^{*}$ & $17.2 \pm 10.1$ & $19.5 \pm 8.2^{*}$ & $16.6 \pm 8.9$ & $20.7 \pm 7.5^{*}$ \\
\hline \multicolumn{2}{|l|}{$\begin{array}{l}\text { Normal walking for } \\
30 \mathrm{~m}(\mathrm{~s})\end{array}$} & $21.1 \pm 3.0$ & $20 \pm 3.1^{*}$ & $21.4 \pm 2.9$ & $20.7 \pm 3.2$ & $21.3 \pm 2.9$ & $20.5 \pm 3.0^{*}$ \\
\hline \multicolumn{2}{|l|}{$\begin{array}{l}\text { Maximum walking } \\
\text { for } 30 \mathrm{~m}(\mathrm{~s})\end{array}$} & $15.9 \pm 3.9$ & $15.7 \pm 3.6$ & $17.1 \pm 3.7 \#$ & $16.9 \pm 3.6 \#$ & $16.6 \pm 3.3$ & $16.4 \pm 3.1$ \\
\hline \multicolumn{2}{|l|}{ Rising (s) } & $3.5 \pm 1.4$ & $3.1 \pm 1.2^{*}$ & $3.9 \pm 1.1 \#$ & $3.4 \pm 1.2^{*}$ & $3.7 \pm 1.2$ & $3.3 \pm 1.1^{*}$ \\
\hline \multirow{4}{*}{$\begin{array}{l}\text { Bone mineral } \\
\text { content }(\mathrm{kg})\end{array}$} & Trunk & $0.38 \pm 0.07$ & $0.38 \pm 0.07$ & $0.35 \pm 0.08$ & $0.35 \pm 0.08$ & $0.36 \pm 0.07$ & $0.36 \pm 0.08$ \\
\hline & Arms & $0.20 \pm 0.05$ & $0.20 \pm 0.05$ & $0.19 \pm 0.04 \#$ & $0.19 \pm 0.03 \#$ & $0.19 \pm 0.04$ & $0.19 \pm 0.04$ \\
\hline & Legs & $0.55 \pm 0.13$ & $0.55 \pm 0.13$ & $0.52 \pm 0.09 \#$ & $0.51 \pm 0.09 \#$ & $0.53 \pm 0.11$ & $0.53 \pm 0.11$ \\
\hline & Subtotal & $1.15 \pm 0.18$ & $1.15 \pm 0.17$ & $1.05 \pm 0.18 \#$ & $1.05 \pm 0.19 \#$ & $1.10 \pm 0.19$ & $1.10 \pm 0.19$ \\
\hline \multirow[t]{4}{*}{ Fat mass $(\mathrm{kg})$} & Trunk & $9.05 \pm 3.29$ & $8.57 \pm 3.18^{*}$ & $8.54 \pm 3.37$ & $8.36 \pm 3.20$ & $8.77 \pm 3.32$ & $8.45 \pm 3.17 *$ \\
\hline & Arms & $2.49 \pm 0.89$ & $2.46 \pm 0.91$ & $2.37 \pm 0.72$ & $2.36 \pm 0.77$ & $2.42 \pm 0.80$ & $2.41 \pm 0.83$ \\
\hline & Legs & $5.89 \pm 2.08$ & $5.75 \pm 2.15^{*}$ & $5.80 \pm 1.53$ & $5.64 \pm 1.49^{*}$ & $5.84 \pm 1.79$ & $5.69 \pm 1.81^{*}$ \\
\hline & Subtotal & $17.67 \pm 5.47$ & $17.02 \pm 5.53^{*}$ & $16.71 \pm 5.12$ & $16.36 \pm 4.93 *$ & $17.15 \pm 5.27$ & $16.66 \pm 5.18^{*}$ \\
\hline \multirow{4}{*}{$\begin{array}{l}\text { Lean soft tissue } \\
\text { mass }(\mathrm{kg})\end{array}$} & Trunk & $19.01 \pm 2.17$ & $19.24 \pm 2.32 *$ & $18.67 \pm 2.26$ & $18.83 \pm 2.10$ & $18.83 \pm 2.21$ & $19.02 \pm 2.19 *$ \\
\hline & Arms & $3.69 \pm 0.85$ & $3.77 \pm 0.83$ & $3.64 \pm 0.47$ & $3.68 \pm 0.44$ & $3.66 \pm 0.67$ & $3.73 \pm 0.65$ \\
\hline & Legs & $11.23 \pm 2.31$ & $11.39 \pm 2.24$ & $11.30 \pm 1.33$ & $11.28 \pm 1.21$ & $11.27 \pm 1.84$ & $11.33 \pm 1.75$ \\
\hline & Subtotal & $34.37 \pm 3.74$ & $34.85 \pm 3.58^{*}$ & $33.61 \pm 3.61$ & $33.80 \pm 3.40$ & $33.95 \pm 3.66$ & $34.28 \pm 3.50 *$ \\
\hline Body mass (kg) & Subtotal & $53.20 \pm 8.37$ & $53.02 \pm 8.45$ & $51.37 \pm 7.99$ & $51.21 \pm 7.75$ & $52.20 \pm 8.16$ & $52.03 \pm 8.07$ \\
\hline $\begin{array}{l}\text { Subcutaneous fat } \\
\left(\mathrm{cm}^{2}\right)\end{array}$ & & $219.0 \pm 71.4$ & $206.6 \pm 68.3^{*}$ & $194.7 \pm 79.2$ & $189.2 \pm 80.2$ & $208.0 \pm 73.1$ & $199.8 \pm 72.0^{*}$ \\
\hline Visceral fat $\left(\mathrm{cm}^{2}\right)$ & & $142.6 \pm 46.8$ & $138.6 \pm 47.4$ & $154.4 \pm 60.9$ & $149.7 \pm 65.5$ & $150.8 \pm 52.4$ & $146.5 \pm 55.7$ \\
\hline
\end{tabular}

Data are means \pm SD

* $P<0.05$ vs. baseline, \# $P<0.05$ vs. $60-69$ years group at baseline or post-intervention

Table 3 Characteristics and anthropometry of the second study participants

\begin{tabular}{lcr}
\hline & $\begin{array}{c}\text { Qigong and } \\
\text { aerobic }(n=29)\end{array}$ & \multicolumn{1}{c}{$\begin{array}{l}\text { Qigong } \\
(n=29)\end{array}$} \\
\hline Age (year) & $67.0 \pm 1.3$ & $67.5 \pm 2.0$ \\
Height $(\mathrm{cm})$ & $150.8 \pm 6.3$ & $151.9 \pm 5.3$ \\
Weight $(\mathrm{kg})$ & $53.2 \pm 8.9$ & $56.5 \pm 9.2$ \\
Body mass index $\left(\mathrm{kg} / \mathrm{m}^{2}\right)$ & $23.4 \pm 3.7$ & $24.4 \pm 3.5$ \\
\hline
\end{tabular}

Data are means \pm SD

Daily moderate or high-intensity exercise substantially reduces body fat [20-23]. Regular moderate exercises, even when not associated with weight loss, reduce serum triglyceride and abdominal subcutaneous and visceral fat in both middle aged and elderly obese subjects with insulin resistance [20, 23]. Furthermore, such exercises also increase lean body mass and total skeletal muscle [20, 23]. The qigong and aerobic exercise program here was associated with a reduction of fat mass and an increase of lean soft tissue without significantly changing subtotal body mass, although the intensity of qigong and aerobic exercise seems to be much lower than regular endurance training that can reduce body fat and increase lean body mass. The 12-week exercise program was associated with a decrease of subcutaneous abdominal fat but not visceral abdominal fat.

Loading influences bone mass, micro-architecture, and size throughout life [24]. Bone mass is continuously lost from 25-30 years of age [24]. Exercise (loading) appears to be important for maintenance of bone mass during aging. However, reported effects of exercise on prevention of bone loss have not always been consistent [25, 26]. A review article [26] presented the positive effects of exercise training programs for prevention of bone loss in pre and 
Table 4 Comparison between qigong and aerobic exercise and qigong exercise alone in effects on physical function

\begin{tabular}{|c|c|c|c|c|c|c|c|}
\hline & \multicolumn{2}{|c|}{ Qigong and aerobic $(n=29)$} & \multicolumn{2}{|c|}{ Qigong $(n=29)$} & \multicolumn{3}{|c|}{ Interaction between group and time } \\
\hline & Baseline & Post-intervention & Baseline & Post-intervention & $\begin{array}{l}\text { Degrees } \\
\text { of freedom }\end{array}$ & $F$ values & $P$ values \\
\hline Normal walking for $30 \mathrm{~m}(\mathrm{~s})$ & $21.3 \pm 1.9$ & $19.6 \pm 1.8^{*}$ & $21.2 \pm 1.9$ & $19.7 \pm 1.8^{*}$ & 1 & 0.01 & 0.92 \\
\hline Maximum walking for $30 \mathrm{~m}$ (s) & $17.1 \pm 1.4$ & $15.8 \pm 1.1^{*}$ & $17.5 \pm 1.7$ & $16.6 \pm 1.7 *$ & 1 & 0.01 & 0.91 \\
\hline Rising (s) & $3.9 \pm 0.8$ & $3.2 \pm 0.8^{*}$ & $3.9 \pm 0.8$ & $3.3 \pm 0.9^{*}$ & 1 & 0.02 & 0.89 \\
\hline
\end{tabular}

Data are means $\pm \mathrm{SD}$

$* P<0.05$ vs. baseline

postmenopausal women, but another study [26] did not support the effects in pre-menopausal women. The qigong and aerobic exercise program did not alter the BMC in the trunk, arms, legs, or subtotal body during the 12 weeks. The $\mathrm{BMC}$ results in this study suggest that our 12-week qigong and aerobic exercise program in elderly women did not have a positive association for prevention of bone loss.

In our second study, similar to the qigong and aerobic exercise program, the program of qigong exercise alone demonstrated positive changes on physical function in elderly women. We could not find any significant differences in age, anthropometric features, or physical function at baseline between the two groups. Thus, we believe that the two groups ("the qigong and aerobic" group and "qigong alone" group) were divided evenly, although the participants in the study were not assigned to groups randomly but according to their residences.

In the first study, the 12-week qigong and aerobic exercise program was associated with improvements in physical function and a reduction in body fat in elderly women. However, it was unclear how much each exercise ("qigong" or "aerobic") contributed to the positive changes during the 12-week program. The optional aerobic exercise programs offered once a week might have positively influenced physical function and body fat. However, the 12-week program was mainly composed of qigong exercise, as was the daily home qigong practice. In the second study, the qigong exercise alone positively influenced physical function similar to the combined qigong and aerobic exercise program. Still, we believe that the qigong practice was largely responsible for the association with improvements in physical function and a reduction of body fat observed in the elderly women.

The qigong and aerobic exercise program was well received overall. There were no accidents such as falls or aggravation of symptoms during the different sessions of the 12-week program. Ninety-five percent of the participants who completed similar exercise programs in our other studies $(n=105)$ indicated in their exit questionnaires that they would continue the qigong practice at home or participate in the session program.
Physical activity programs designed for the elderly should always be cardiovascularly safe. We did not record physiological signals to monitor cardiovascular function such as blood pressure or heart rate during the qigong training. However, the qigong training in this study appeared to have a low risk of causing cardiovascular problems because of its unique training style and slow whole-body performance with natural breathing.

Controlled trials investigating qigong programs without any optional exercises are needed to confirm the positive changes found in this exploratory study. The safety of the program with regard to cardiovascular function during training must also be demonstrated. Follow-up studies should also be conducted to determine if the positive changes in physical function and body fat can be sustained over time in the elderly.

\section{Conclusion}

The findings of this exploratory study demonstrated that a 12 -week qigong and aerobic exercise program was associated with improvements in physical function and a reduction in body fat. The qigong exercise program alone positively influenced physical function. The qigong program appears to be an appealing means to improve the physical health of elderly persons.

Acknowledgments The authors thank Dr Toshikazu Kitade (Kitade Hospital, Gobo, Japan) for valuable advice during this study.

\section{References}

1. Tsang HW, Mok CK, Au Yeung YT, Chan SY. The effect of Qigong on general and psychosocial health of elderly with chronic physical illnesses: a randomized clinical trial. Int $\mathbf{J}$ Geriatr Psychiatry. 2003;18:441-9.

2. Lee MS, Jeong SM, Oh SW, Ryu H, Chung HT. Effects of chundosunbup Qi-training on psychological adjustments: a crosssectional study. Am J Chin Med. 1998;26:223-30.

3. Lee MS, Lee MS, Kim HJ, Moon SR. Qigong reduced blood pressure and catecholamine levels of patients with essential hypertension. Int J Neurosci. 2003;113:1691-701. 
4. Lee MS, Kang CW, Ryu H, Kim JD, Chung HT. Effects of ChunDoSunBup Qi-training on growth hormone, insulin-like growth factor-I, and testosterone in young and elderly subjects. Am J Chin Med. 1999;27:167-75.

5. Lee MS, Kang CW, Shin YS, Huh HJ, Ryu H, Park JH, et al. Acute effects of chundosunbup qi-training on blood concentrations of TSH, calcitonin, PTH and thyroid hormones in elderly subjects. Am J Chin Med. 1998;26:275-81.

6. Ryu H, Jun CD, Lee BS, Choi BM, Kim HM, Chung HT. Effect of qigong training on proportions of $\mathrm{T}$ lymphocyte subsets in human peripheral blood. Am J Chin Med. 1995;23: 27-36.

7. Kuo TC, Ho FM, Lin CW. Physiological changes associated with Neiyang Qigong. J Health Sci. 1993;49:278-84.

8. Li Q, Matsuura Y, Tanaka Y, Tsubouchi S, Li Q, Shimizu N. Effects of Shaolin Internal Qigong on physiological changes. J Acupunct Tuina Sci. 2004;2:57-60.

9. Suzuki T, Kim H, Yoshida H, Ishizaki T. Randomized controlled trial of exercise intervention for the prevention of falls in community-dwelling elderly Japanese women. J Bone Miner Metab. 2004;22:602-11.

10. Irwin ML, Yasui Y, Ulrich CM, Bowen D, Rudolph RE, Schwartz RS, et al. Effect of exercise on total and intra-abdominal body fat in postmenopausal women: a randomized controlled trial. JAMA. 2003;289:323-30.

11. Cress ME, Buchner DM, Prohaska T, Rimmer J, Brown M, Macera $\mathrm{C}$, et al. Best practices for physical activity programs and behavior counseling in older adult populations. J Aging Phys Act. 2005; 13:61-74.

12. Wu G. Evaluation of the effectiveness of Tai Chi for improving balance and preventing falls in the older population: a review. J Am Geriatr Soc. 2002;50:746-54.

13. Wang C, Collet JP, Lau J. The effect of Tai Chi on health outcomes in patients with chronic conditions: a systematic review. Arch Int Med. 2004;164:493-501.

14. Hogan M. Physical and cognitive activity and exercise for older adults: a review. Int J Aging Hum Dev. 2005;60:95-126.

15. Hunter GR, McCarthy JP, Bamman MM. Effects of resistance training on older adults. Sports Med. 2004;34:329-48.
16. Latham N, Anderson C, Bennett D, Stretton C. Progressive resistance strength training for physical disability in older people. Cochrane Database Syst Rev. 2003;CD002759.

17. Van Pelt RE, Evans EM, Schechtman KB, Ehsani AA, Kohrt WM. Contributions of total and regional fat mass to risk for cardiovascular disease in older women. Am J Physiol Endocrinol Metab. 2002;282:E1023-8.

18. Tanko LB, Bagger YZ, Qin G, Alexandersen P, Larsen PJ, Christiansen C. Enlarged waist combined with elevated triglycerides is a strong predictor of accelerated atherogenesis and related cardiovascular mortality in postmenopausal women. Circulation. 2005;111:1883-90.

19. Nagasaki H, Itoh H, Furuna T. A physical fitness model of older adults. Aging (Milano). 1995;7:392-7.

20. Lee S, Kuk JL, Davidson LE, Hudson R, Kilpatrick K, Graham TE, et al. Exercise without weight loss is an effective strategy for obesity reduction in obese individuals with and without Type 2 diabetes. J Appl Physiol. 2005;99:1220-5.

21. Ross R, Janssen I, Dawson J, Kungl AM, Kuk JL, Wong SL, et al. Exercise-induced reduction in obesity and insulin resistance in women: a randomized controlled trial. Obes Res. 2004;12:789-98.

22. Wilmore JH, Despres JP, Stanforth PR, Mandel S, Rice T, Gagnon $\mathrm{J}$, et al. Alterations in body weight and composition consequent to $20 \mathrm{wk}$ of endurance training: the HERITAGE Family Study. Am J Clin Nutr. 1999;70:346-52.

23. Lehmann R, Vokac A, Niedermann K, Agosti K, Spinas GA. Loss of abdominal fat and improvement of the cardiovascular risk profile by regular moderate exercise training in patients with NIDDM. Diabetologia. 1995;38:1313-9.

24. Mosekilde L. Age-related changes in bone mass, structure, and strength-effects of loading. Z Rheumatol. 2000;59(Suppl 1):1-9.

25. Wolff I, van Croonenborg JJ, Kemper HC, Kostense PJ, Twisk JW. The effect of exercise training programs on bone mass: a meta-analysis of published controlled trials in pre- and postmenopausal women. Osteoporos Int. 1999;9:1-12.

26. Kelley GA, Kelley KS. Efficacy of resistance exercise on lumbar spine and femoral neck bone mineral density in premenopausal women: a meta-analysis of individual patient data. J Womens Health (Larchmt). 2004;13:293-300. 